\title{
Resonant Transmission of Magneto-atmospheric Waves in Sunspot Umbrae
}

\author{
J. Staude, J. Rendtel and A. Settele \\ Astrophysikalisches Institut Potsdam, Sonnenobservatorium \\ Einsteinturm, 14473 Potsdam, Germany
}

\begin{abstract}
We discuss intensity and velocity oscillations which were observed by SOHO in lines of C IV and Ne VIII formed in the EUV plume above a sunspot during a continuous time series of 4.3 hours. Applying a wavelet analysis, we find periods mainly around $3 \mathrm{mHz}$; the behavior in time is different for the different EUV lines and strongly intermittent, for the velocity oscillations in particular. Moreover, we discuss the observed oscillations within the frame of model calculations for the resonant transmission of magneto-atmospheric waves through realistic models of the sunspot atmosphere. The nonstationary behavior could hint at the appearance of blinkers in the umbral transition region.
\end{abstract}

\section{Introduction}

The sharp resonance peaks of the power of oscillations of velocity $\delta v$ and intensity $\delta I$, observed in lines and continua in sunspots, in the umbra in particular, show a characteristic height dependence of frequencies and phases. The peaks are closely packed and concentrated in several period bands: at $2 \ldots 3 \mathrm{~min}$, $5 \mathrm{~min}$, and $\gtrsim 20 \mathrm{~min}$. The $20 \mathrm{~min}$ and 5 min periods dominate in the photosphere, the $2 \ldots 3$ min periods in the chromosphere and transition region to the corona (CCTR). These features can be explained by the resonant transmission of magneto-atmospheric waves (magneto-acoustic gravitational or MAG waves) through realistic models of a stratified sunspot umbra atmosphere. Such models of coupled resonators require regions of strong wave reflection, e.g. from the steep temperature gradient of the CCTR. Consequently, oscillations should be observed in the EUV lines from the sunspot CCTR as well. A review on observations and theoretical modeling of sunspot oscillations has recently been given by Staude (1999).

\section{Observations}

Significant oscillations of $\delta v$ and $\delta I$ in the CCTR above sunspots have been discovered by Gurman et al. (1982) with the UVSP instrument aboard the SMM spacecraft. The present investigation aims at a deeper insight into such processes, having available more complete data, that is, longer time series, better spatial resolution, and simultaneous measurements in several CCTR lines, including those corresponding to higher temperatures. 

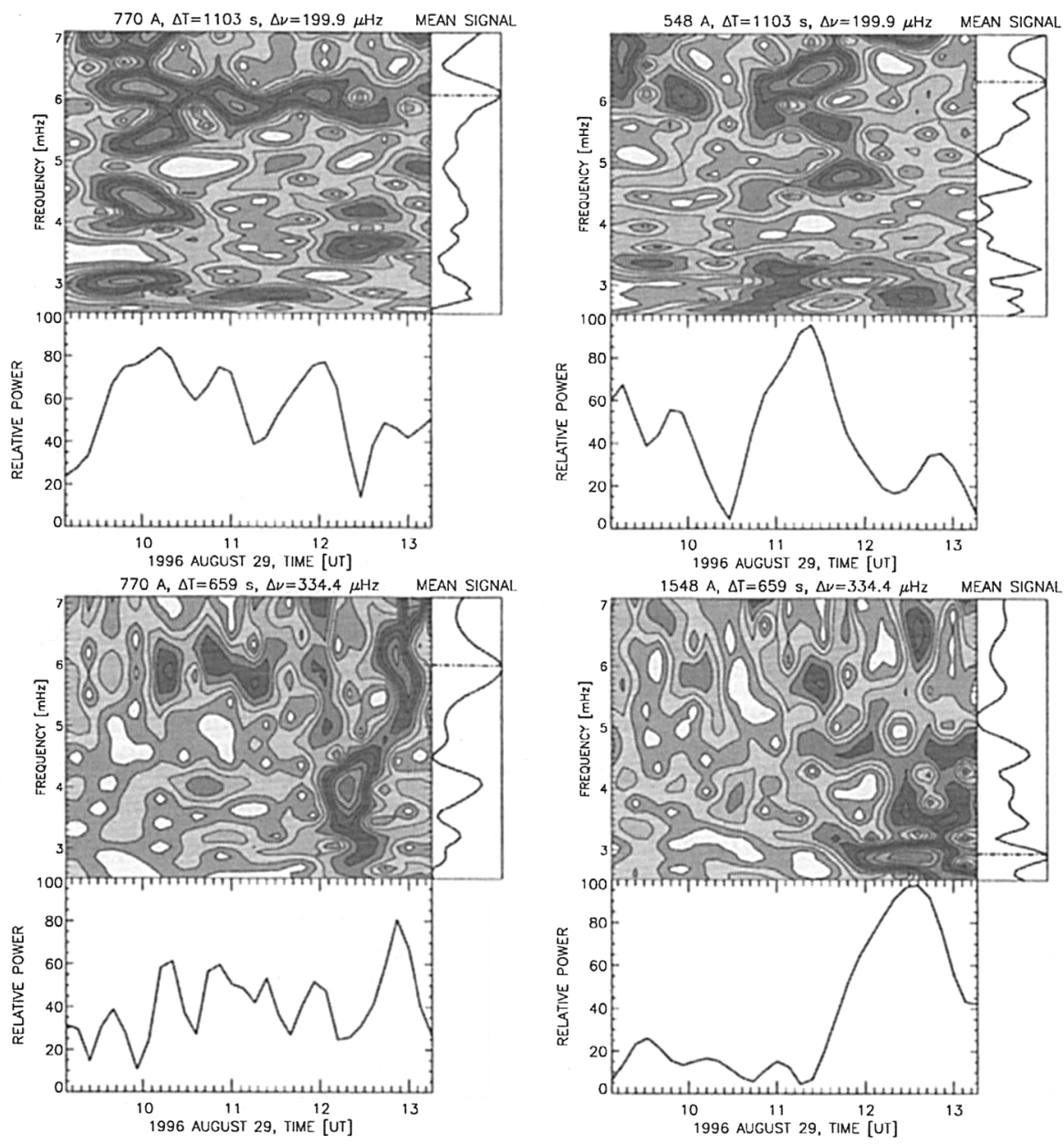

Figure 1. Wavelet analysis of a time series obtained with SUMER from a large sunspot with an EUV plume in NOAA 7986 on Aug. 29, 1996, 09:08-13:31 UT. During this time, scans were made continuously over the active region which was not far from the center of the solar disk. The pictures show relative power (maximum power within the frequency-time space set to 100) of intensity $\delta I$ (top) and of velocity oscillations $\delta v$ (bottom) in the lines Ne VIII $770.4 \AA\left(T \approx 6 \times 10^{5} \mathrm{~K}\right)$ and C IV $1548.1 \AA\left(T \approx 10^{5} \mathrm{~K}\right)$, respectively, integrated over the umbral core to improve the signal-to-noise ratio. The additional panels show integrated power over frequency (right) and the temporal variations of power at the frequency with the highest mean signal (lower panels). 

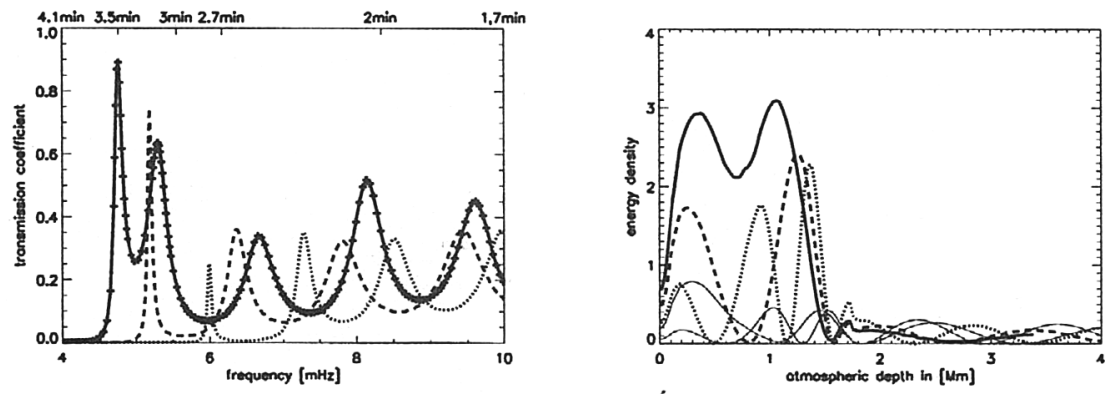

Figure 2. Left: resonant transmission of MAG waves through the umbral model of Staude (1981) and Obridko \& Staude (1988), solid curve: for realistic $\gamma(z)$ and $P_{t u}(z)$, dashed: for constant $\gamma$, dotted: for constant $\gamma$ and $P_{t u}$. Right: energy density in the model of the preceding figure with $\gamma(z)$ and $P_{t u}(z)$ for different resonant frequencies (thick curves) and intermediate frequencies (thin curves). $z=0$ is fixed at the position of the CCTR; the lower photosphere (optical depth $\left.\tau_{0}=1\right)$ is at a geometrical depth of $z=1.8 \times 10^{6} \mathrm{~m}$.

Here we present observations with the SUMER spectrograph aboard SOHO. The longest time series for our purpose was obtained on August 29, 1996 (Rendtel et al. 1998) in lines of C IV and Ne VIII. Details of the observing procedure and results of the analysis are given in Fig. 1.

Maximum power in $\delta I$ is close to a period of $3 \mathrm{~min}$, with strong variations in time. Both lines show similar power periods but with a tendency to an opposite behavior in time. We also find power in $\delta I$ near $137 \mathrm{~s}(\hat{=} 7.5 \mathrm{mHz})$ which is close to the Nyquist frequency $(7.6 \mathrm{mHz})$, but several properties suggest a real umbral origin: concentration of power only in the line and only in the umbra, different behavior of both lines in time (see the upper edge of the respective diagrams in Fig. 1). Simultaneous EIT observations (09:00-11:00 UT) in Fe IX/X at $171 \AA$ $\left(T \approx 10^{6} \mathrm{~K}\right)$ do not show any prominent $\delta I$ periods.

In $\delta v$ the power is more intermittent. The maximum in Ne vIII is at $6 \mathrm{mHz}$, that is close to the same frequency as in $\delta I$. In C IV lower frequencies dominate for $\delta v$. The oscillations occur highly variable, especially between 12 and $13 \mathrm{UT}$. In this interval we find a prominent occurrence of a 5 -min oscillation $(3.0 \mathrm{mHz})$ in the CIV line, and at the same time a rapid drift of the peak frequency of $\delta v$ from $4 \mathrm{mHz}$ to $6 \mathrm{mHz}$ in the (higher) Ne VIII line. This coincides with changes in the oscillation pattern in $\delta I$ observed after $12 \mathrm{UT}$, although there is no obvious change in the spot as derived from EIT or reconstructed SUMER images.

\section{Modeling}

A first successful approach to model sunspot oscillations by the resonant transmission of MAG waves is due to Zhugzhda et al. (1983, 1984, 1987), Gurman \& Leibacher (1984), and Staude et al. (1985); these calculations were limited to pure vertical oscillations. The theory has now been generalized by Settele 
et al. (1999) by applying a new approach to include horizontal oscillations as well. The results depend critically on the model atmosphere, thereby providing a basis for sunspot seismology. The dependence of both the adiabatic coefficient $\gamma$ and the turbulent pressure $P_{t u}$ on depth $z$ must carefully be taken into account - contrary to a wide-spread practice (see Fig. 2). The model calculations are able to reproduce some basic features of the observed sunspot oscillations (multiple-peak structure of the oscillatory power spectrum, height dependence of amplitudes and phases of $\delta I$ and $\delta v$ fluctuations up to the CCTR), but not the intermittent behavior: maybe the measurements are influenced by such phenomena as blinkers which have time scales similar to those of the nonstationary behavior in the umbral oscillations. EUV blinkers have been observed not only above the supergranular network, but above sunspots too (see the contribution by Parnell, Brewsher, Harrison, \& Hood in the present volume).

Acknowledgments. The present work has been supported by the German Science Foundation (DFG) through the grants STA 351/4-2 and Sta 351/5-3. We are grateful to the SUMER team, in particlar to D. Innes, C.-R. de Boer, and K. Wilhelm, for supporting our SUMER observations. J.B. Gurman kindly provided the simultaneous EIT data for comparison. The SUMER instrument is financially supported by DLR, CNES, NASA, and ESA. It is part of SOHO, the Solar and Heliospheric Observatory, of ESA and NASA.

\section{References}

Gurman, J.B. \& Leibacher, J.W. 1984, ApJ, 283, 859

Gurman, J.B., Leibacher, J.W., Shine, R.A., Woodgate, B.E., \& Henze, W. 1982, ApJ, 253, 939

Rendtel, J., Staude, J., Innes, D., Wilhelm, K., \& Gurman, J.B. 1998, ESA SP-417, 277

Settele, A., Zhugzhda, Y.D., \& Staude, J. 1999, Astron. Nachr., 320, 147

Staude, J. 1981, A\&A, 100, 284

Staude, J. 1999, in ASP Conf. Ser. Vol. 184, Magnetic Fields and Oscillations. 3rd Adv. in Solar Physics Euroconf., ed. B. Schmieder, A. Hofmann \& J. Staude (San Francisco: ASP), 113.

Staude, J., Zhugzhda, Y.D., \& Locāns, V. 1985, Solar Phys., 95, 37

Zhugzhda, Y.D., Locāns, V., \& Staude, J. 1983, Solar Phys., 82, 369

Zhugzhda, Y.D., Locāns, V., \& Staude, J. 1987, Astron. Nachr., 308, 257

Zhugzhda, Y.D., Staude, J., \& Locāns, V. 1984, Solar Phys., 91, 219 